\title{
Inhibiting Necroptosis of Spermatogonial Stem Cell as a Novel Strategy for Male Fertility Preservation
}

\author{
Yun Xie, ${ }^{1-3,{ }^{*}}$ Haicheng Chen,, ${ }^{1, *}$ Daosheng Luo, ${ }^{4}$ Xing Yang, ${ }^{2}$ Jiahui Yao, ${ }^{1}$ Chi Zhang, ${ }^{1}$ Linyan Lv, 2,5 \\ Zexin Guo, ${ }^{1}$ Cuncan Deng, ${ }^{2,5}$ Yanqing Li, ${ }^{2,5}$ Xiaoyan Liang, ${ }^{2}$ Chunhua Deng, ${ }^{1}$ \\ Xiangzhou Sun, and Guihua Liu²
}

Fertility preservation is a common concern for male cancer survivors of reproductive age. However, except for testicular tissue cryopreservation, which is not very effective, there is no feasible and precise therapy capable of protecting spermatogenesis for prepubertal boys before or during gonadotoxic treatment. This study aims to investigate the effects of inhibiting necroptosis of spermatogonial stem cell (SSC) in fertility preservation. Male mice 12 weeks of age were used to establish gonadotoxicity with two intraperitoneal injections of busulfan at a total dose of $40 \mathrm{mg} \mathrm{kg}^{-1}$. The mouse model and the primary cultured mouse SSCs were used to characterize the relationship between necroptosis of SSC and gonadotoxicity. Meanwhile, the effects of an inhibitor of necroptosis pathway, RIPA-56, were observed on day 36 in the mouse model of busulfan-induced gonadotoxicity. We found that the number of SSCs was decreased, but the level of necroptosis was upregulated on day 18 after busulfan treatment in testes from gonadotoxic mice. Further experiments in primary cultured cells showed that the necroptosis caused cell death in busulfan-treated SSCs and could be inhibited by RIPA-56. After suppressing the necroptosis of SSCs, the busulfan-induced mice had a decreased loss of spermatogenic cells as shown by histology and an increased Johnsen's score. Moreover, the quantities of SSCs and epididymal spermatozoa were restored after intervention with RIPA-56, indicating a series of beneficial effects by targeting the necroptosis of SSCs in mice undergoing busulfan treatment. In conclusion, our findings reveal that the necroptosis of SSCs plays a critical role in busulfan-induced gonadotoxicity and may be a potential target for male fertility preservation.

Keywords: busulfan, fertility preservation, gonadotoxicity, necroptosis, spermatogonial stem cell

\section{Introduction}

$\mathrm{C}$ ANCER INCIDENCE IS increasing rapidly each year and there were an estimated 18.1 million new cancer cases in 185 countries in 2018 [1]. Treatments for neoplastic disease, including radiotherapy, chemotherapy, and combinations of these therapies, may lead to male gonadotoxic side effects, which result in loss of fertility, particularly in young cancer survivors, further impacting quality of life [2]. Because germ cells are extremely sensitive to radiation and chemotherapeutic toxicity [3], prolonged and permanent azoospermia often occur after cancer treatment [4]. Guidelines published by the American Society of Clinical Oncology (ASCO) highly recommend that sperm cryopreservation is effective, and health care providers should discuss sperm banking with postpubertal males receiving cancer treatment [5]. However, there are no proven fertility preservation options for prepubertal patients not yet producing mature sperm currently [6]. Some drug therapies are ever used to preserve male fertility by reducing or blocking the gonadotoxic impact of chemotherapy or radiation on the testes [7]. Unfortunately, hormone injections to preserve fertility have not proven to be useful in males, and antiapoptotic agents such as sphingosine-1phosphate have been shown to have limited value $[8,9]$. Moreover, the cytoprotective agents or granulocyte colonystimulating factor were applied to protect male fertility in those who were undergoing chemotherapy, but the effects need to be further confirmed [10-13]. In addition, stem cellbased options to preserve male fertility still have a long way to go due to their complexity $[14,15]$. Consequently, there is

\footnotetext{
${ }^{1}$ Department of Andrology, The First Affiliated Hospital, Sun Yat-sen University, Guangzhou, China.

${ }^{2}$ Reproductive Centre, The Sixth Affiliated Hospital, Sun Yat-sen University, Guangzhou, China.

${ }^{3}$ Guangdong Provincial Key Laboratory of Orthopedics and Traumatology, Guangzhou, China.

${ }^{4}$ Department of Urology, Southern Medical University Affiliate Dongguan People's Hospital, Dongguan, China.

${ }^{5}$ Gastrointestinal Diseases Research Institute of Guangdong Province, The Sixth Affiliated Hospital, Sun Yat-sen University, Guangzhou, China.

*These authors contributed equally to this work.
} 
still no feasible and precise method for protecting spermatogenesis in prepubertal male cancer patients before or during gonadotoxic treatment.

Alkylating agents are commonly used in cancer treatment. Busulfan is a classic alkylating agent that severely damages spermatogonial stem cells (SSCs) and spermatogenesis [16]. Therefore, the mouse model of busulfan-induced gonadotoxicity is a powerful tool for understanding the mechanism of chemotherapy-induced gonadotoxicity and its exploration of treatment $[17,18]$. Necroptosis, a form of regulated necrotic cell death mediated by receptor-interacting protein kinase 1 (RIPK1), receptor-interacting protein kinase 3 (RIPK3) activity, and mixed lineage kinase domain like (MLKL) [19,20], was detected specifically in SSCs in the testes of old mice and proven to promote aging-associated deterioration of the male reproductive system [21]. Furthermore, suppressing necroptosis by a RIPK1 inhibitor was demonstrated as an apparently effective way to delay the age-related changes in reproductive organs [21]. However, whether the necroptosis is associated with chemotherapy-related SSCs and spermatogenesis damage and whether a RIPK1 inhibitor will protect the testes from chemotherapeutic toxicity remain to be elucidated.

Thus, this study was designed to analyze how necroptosis works in a mouse model of busulfan-induced gonadotoxicity and to investigate whether inhibition of the RIPK1-driven necroptosis pathway could be a novel strategy for male fertility preservation.

\section{Materials and Methods}

\section{Animal use and care}

The C57BL/6J male mice 12 weeks of age (25-35 g), Institute of Cancer Research (ICR) mice, and Kunming male mice were purchased from the Animal Laboratory Center of Sun Yat-sen University (Guangzhou, Guangdong, China) and were housed under specific pathogen-free conditions. The animal procedures were approved by the Institutional Animal Care and Use Committee (IACUC) of Sun Yat-sen University (SYSU-IACUC-2019-000086).

\section{Animal model}

The mouse model of busulfan-induced gonadotoxicity was generated by two intraperitoneal injections of busulfan (No. HY-B0245; MedChemExpress, NJ) at a total dose of $40 \mathrm{mg}$ $\mathrm{kg}^{-1}$ body weight with an interval of $3 \mathrm{~h}$ as we previously described [17]. According to the seminiferous epithelium cycle in mice (four stages, 8.6 days per stage) [22], mice were randomly euthanatized at different time points after busulfan treatment to analyze spermatogenesis alterations in testes.

\section{Administration of RIPK1 inhibitor RIPA-56}

RIPA-56 (No. HY-101032; MedChemExpress) was prepared fresh before every use. In total, $2 \mathrm{mg}$ RIPA-56 was placed into a $1.5 \mathrm{~mL}$ centrifuge tube, $120 \mu \mathrm{L}$ dimethyl sulfoxide (DMSO) was immediately added, and the mixture was shaken rigorously. The dissolved RIPA-56 was diluted with $1.08 \mathrm{~mL}$ normal saline to make the final working solution. Finally, this diluted RIPA-56 was administered intragastrically at $60 \mathrm{mg} \mathrm{kg}^{-1}$ body weight every day [21].

\section{Testis weight and histological evaluation}

The testes were weighed at the time of euthanasia and fixed in $4 \%$ paraformaldehyde for $24 \mathrm{~h}$, dehydrated, and embedded in paraffin for histological evaluation. Five-micron sections were cut, and hematoxylin and eosin $(\mathrm{H} \& \mathrm{E})$ staining was performed to observe the structure of seminiferous tubules using a microscope (BX53; OLYMPUS, Tokyo, Japan). Histological evaluation (Johnsen's score) of all mouse testicular tissues was carried out according to a standardized procedure [23].

\section{Epididymal spermatozoa count}

After sacrificing the mice, freshly isolated cauda epididymidis samples that were cleaned of small blood vessels and fat were immediately placed in a cell culture dish containing $0.5 \mathrm{~mL} 4 \%$ bovine serum albumin (BSA) (No. A1933; Sigma, St. Louis, MO) in Biggers, Whitten, and Whittingham medium (Irvine Scientific, Santa Ana, CA). The cauda epididymidis was cut into small pieces and incubated at $37^{\circ} \mathrm{C}$ for $10 \mathrm{~min}$. The total spermatozoa count was evaluated using the Computer-Assisted Semen Analysis system (Hamilton Thorne, Beverly, MA).

\section{Isolation and culture of primary mouse SSCS}

To derive SSCs, the Kunming male mice (at postnatal day 10) were sacrificed for testes. The testes were digested with $1 \mathrm{mg} / \mathrm{mL}$ collagenase IV (No. C5138; Sigma) for $15 \mathrm{~min}$ at $37^{\circ} \mathrm{C}$, followed by $0.05 \%$ trypsin-EDTA (No. 25200-072; Thermo Fisher Scientific, MA) digestion for $10 \mathrm{~min}$ at $37^{\circ} \mathrm{C}$. The testicular cells were collected and plated to $0.1 \%(\mathrm{w} / \mathrm{v})$ gelatin-coated culture dish. Two hours later, the unattached cells were collected and plated to another gelatin-coated culture dish. After three repetitions, the unattached cells were collected and seeded on the feeder cells, cultured with modified SSC medium according to a previously reported protocol [24].

The mouse embryonic fibroblasts (MEFs) were isolated from ICR embryos on day 13.5 postcoitum. The cells at passage 3 were treated with $10 \mu \mathrm{g} / \mathrm{mL}$ mitomycin $\mathrm{C}$ (No. HY-13316; MedChemExpress) for $2 \mathrm{~h}$. After being washed twice with phosphate-buffered saline (PBS), MEFs were plated as feeders for SSCs.

\section{Cell proliferation colorimetric assay}

SSCs with MEFs or MEFs only were seed on 96-well plates at a concentration of 2,000 cells per well in $100 \mu \mathrm{L}$ SSC medium. In tumor necrosis factor-alpha (TNF- $\alpha$ ), SM164, and Z-VAD-FMK (TSZ)-induced assay, cells were treated by different reagents, including PBS, DMSO, and TSZ (Necroptosis Inducer Kit, No. C1058S; Beyotime, Shanghai, China), respectively. According to the protocol of MTS Cell Proliferation Colorimetric Assay kit (No. G3582; Promega, Madison, WI), $20 \mu \mathrm{L}$ MTS reagent was added into each well. After $2 \mathrm{~h}$ of incubation, a microplate reader was used to measure the absorbance at $490 \mathrm{~nm}$. Moreover, to measure the number of SSCs with MEFs or MEFs only, cells of each well were, respectively, treated with $100 \mu \mathrm{M}$ busulfan, $1 \mu \mathrm{M}$ RIPA-56, or $100 \mu \mathrm{M}$ busulfan plus $1 \mu \mathrm{M}$ RIPA-56. Cell proliferation was tested by cell counting kit-8 (CCK-8) assay (No. K1018; ApexBio, Houston, TX), and thereafter, the optical density was measured at $450 \mathrm{~nm}$. 


\section{Western blotting}

The protein concentration was measured by BCA Protein Assay Kit (No. 23227; Thermo Fisher Scientific). Then, these proteins were denatured at $100^{\circ} \mathrm{C}$ for 10 min with $5 \times$ loading buffer. The denatured samples were separated by $10 \%$ sodium dodecyl sulfate-polyacrylamide gel electrophoresis (SDS-PAGE) and transferred onto $0.45 \mu \mathrm{m}$ PVDF membrane (Immobilon-P, IPVH00010; Millipore, MA). Membranes were blocked in TBS-T containing 5\% (v/v) nonfat dry milk (No. NB0669; BBI Life Sciences, Shanghai, China) for $1 \mathrm{~h}$ at room temperature and then incubated overnight at $4^{\circ} \mathrm{C}$ with primary antibodies. After being washed three times with TBS-T, the membranes were incubated with secondary antibodies for $1 \mathrm{~h}$ at room temperature. Following three washes with TBS-T, immunodetection was performed using ChemiDoc Imaging System (Bio-Rad, Hercules, CA). Antibodies used in this study are listed in Supplementary Table S1.

\section{Immunofluorescent staining}

Mouse testicular tissue sections were placed in a $55^{\circ} \mathrm{C}$ incubator and deparaffinized with xylol and different concentrations $(100 \%, 95 \%, 85 \%$, and $75 \%)$ of alcohol $(\mathrm{v} / \mathrm{v})$. Then, these sections were incubated in $3 \% \mathrm{H}_{2} \mathrm{O}_{2}$ to eliminate endogenous peroxidase and subjected to antigen retrieval with Tris-EDTA buffer (pH 9.0) (No. G1203; Servicebio, Wuhan, China). In addition, cultured SSCs were fixed with $4 \%$ paraformaldehyde for $10 \mathrm{~min}$. Subsequently, all sections were washed with PBS for $5 \mathrm{~min}$, permeabilized with $0.1 \%$ Triton $\mathrm{X}-100$ in PBS for $30 \mathrm{~min}$, and then blocked with 5\% BSA in PBS for $10 \mathrm{~min}$. The sections were incubated overnight at $4^{\circ} \mathrm{C}$ with primary antibodies. For a negative control, PBS was used instead of antibody. After being washed with PBS, the sections were incubated with Cy3-conjugated goat anti-rabbit secondary antibody for $1 \mathrm{~h}$ at $37^{\circ} \mathrm{C}$ and washed three times with PBS for $5 \mathrm{~min}$. Nuclei were stained with DAPI $(10 \mu \mathrm{g} / \mathrm{mL}$, No. C0065; Solarbio, Beijing, China) for $7 \mathrm{~min}$ at room temperature. Finally, digital images were obtained by using a fluorescence microscope (IX71; OLYMPUS). Antibodies used in this study are listed in Supplementary Table S1.

\section{Statistical analysis}

All data are presented as the mean \pm standard deviation. Values of $P<0.05$ were considered statistically significant. The differences between two groups were analyzed for statistical significance by using Student's $t$-test. In addition, multigroup comparisons of the means were analyzed by a oneway analysis of variance, followed by a post hoc Bonferroni test. Statistical analysis was performed using SPSS software for Windows, version 20.0 (IBM, Armonk, NY).

\section{Results}

\section{Establishment of the mouse model of busulfan- induced gonadotoxicity}

To explore the chemotherapeutic gonadotoxicity, we built a mouse model with administration of two intraperitoneal injections of busulfan $\left(40 \mathrm{mg} \mathrm{kg}^{-1}\right.$ in total) at a 3 -h interval [17], and observed the dynamic changes of testes in different time points (Fig. 1A). Typically, there were visible histological changes on day 18 , including progressive atrophic lesions, disarrangement of spermatogenic cells, and thinner seminiferous epithelium confirmed by H\&E staining (Fig. 1B). There were no spermatogenic cells, and only Sertoli cells left in seminiferous tubules on day 36 , which is just beyond the time frame of one spermatogenic cycle in mice (34.4 days) [22]. Upon extending the observation to day 63 after busulfan treatment, there were still no spermatogenic cells in seminiferous tubules (Fig. 1B). The expression of ubiquitin C-terminal hydrolase L1 (UCHL1), a marker of SSCs [25-28], in the testes by Western blotting decreased sharply on day 36 compared with day $0(P<0.001$, $n=6$ ) (Fig. 1C). Next, immunofluorescent staining of UCHL1 and alpha-smooth muscle actin ( $\alpha$-SMA), a marker of smooth muscle cells used for identifying contour of seminiferous tubules [17], in testes on day 36 also showed that there were still no SSCs (Fig. 1D). These data supported the idea that gonadotoxicity was elicited in mice by an alkylating agent that is commonly used in chemotherapy. The gonadotoxicity in busulfan-induced mice includes impairment of spermatogenesis and damage to SSCs. Importantly, these endogenous germ cells were destroyed over one spermatogenic cycle and could not recover in a short time, which demonstrated that this busulfan-induced mouse model was suitable for further fertility preservation studies.

\section{The necroptosis of SSCs was closely related to the busulfan-induced gonadotoxicity in mice}

To analyze the role of necroptosis in mice testes with busulfan-induced gonadotoxicity, we evaluated the key components of necroptosis pathway, including TNF- $\alpha$, RIPK1, RIPK3, MLKL, and phosphorylated MLKL (pMLKL), by Western blotting. Interestingly, the necroptosis activation marker, pMLKL, was increased dramatically on day 18 (Fig. 2A), which was consistent with the time of visible histopathological changes and decreased expression of UCHL1. Moreover, with the loss of spermatogenic cells, RIPK3, which is another core component of necroptosis, also declined in testes (Fig. 2A). Further immunofluorescence staining showed that RIPK3 localized in the seminiferous tubules, and pMLKL was detected at the basement membrane in seminiferous tubules on day 18 (Fig. 2B). Based on the above findings, to further determine the relationship between necroptosis and SSCs, we evaluated the kinetic expression of RIPK3, pMLKL, and UCHL1 at 3-day intervals around day 18 (Fig. 2C). On one hand, the ratio of testis weight to body weight decreased gradually with the loss of SSCs (Fig. 2D, E). On the other hand, the appearance of pMLKL in testes accelerated the necroptosis of SSCs, resulting in the decreased expression of UCHL1. Then, with the loss of SSCs, RIPK3 and pMLKL levels also declined (Fig. 2D, E). These data revealed that SSC necroptosis may play a vital role in impairing spermatogenesis of mice with busulfan-induced gonadotoxicity.

\section{The RIPK1-RIPK3-pMLKL pathway mediates necroptosis in mouse SSCS}

To characterize the underlying mechanism causing SSC necroptosis, we performed a series of experiments on SSCs in vitro. The SSCs showed grape-like morphology in vitro 
FIG. 1. Establishment of the mouse model of busulfan-induced gonadotoxicity. (A) The scheme of intraperitoneal injections of busulfan and sample collection time points to investigate the busulfan-induced gonadotoxicity ( $n=6$ per group). (B) The H\&E staining of testes after busulfan treatment. Visible histological changes could be found on day 18 . Scale bar $=100 \mu \mathrm{m}$. (C) The protein expression of UCHL1 in testes after busulfan treatment. Western blotting analysis revealed that there was a decrease on day 36 compared with day $0(* * * P<$ $0.001, n=6$ per group). (D) Immunofluorescent staining of UCHL1 (red) and $\alpha$-SMA (green) in testes after busulfan treatment on day 0 and 36. Scale bar $=75 \mu \mathrm{m}$. H\&E, hematoxylin and eosin; $\alpha$-SMA, alpha smooth muscle actin; UCHL1, ubiquitin C-terminal hydrolase L1.

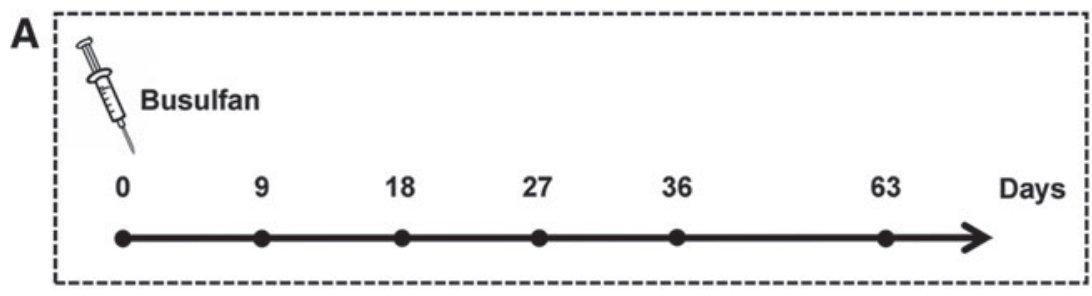

B

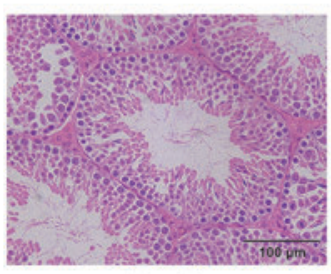

Day 0

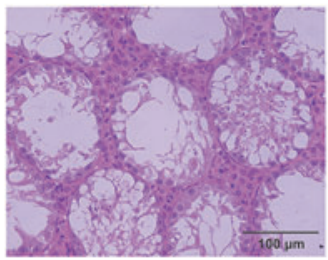

Day 27

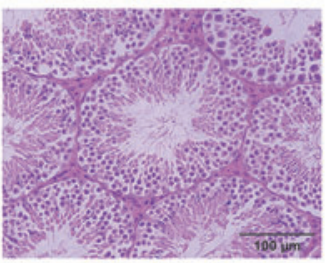

Day 9

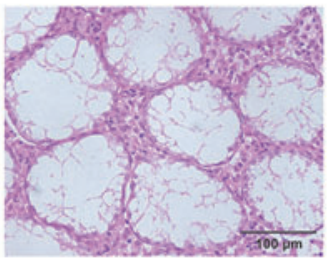

Day 36

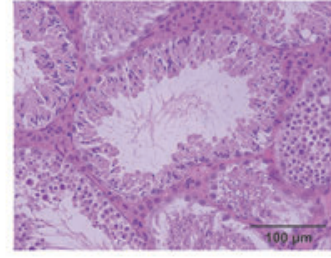

Day 18

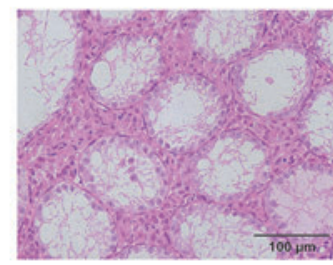

Day 63
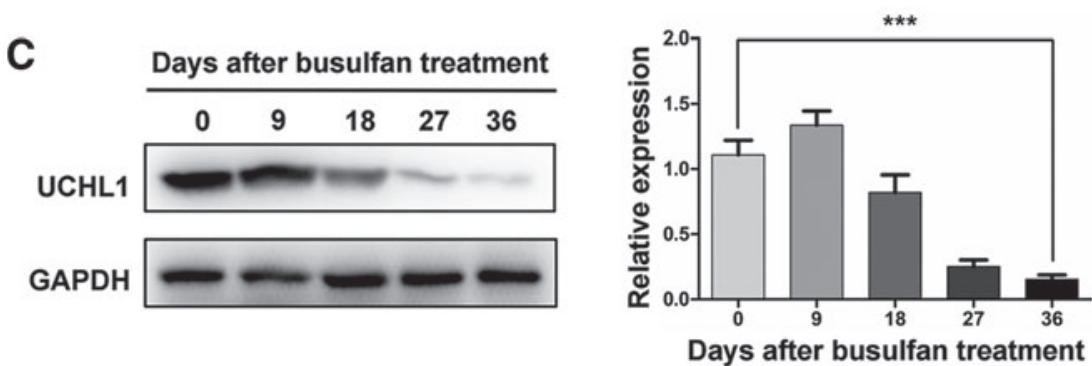

D

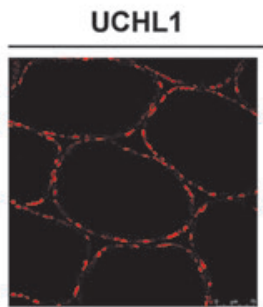

a-SMA

DAPI

Merge
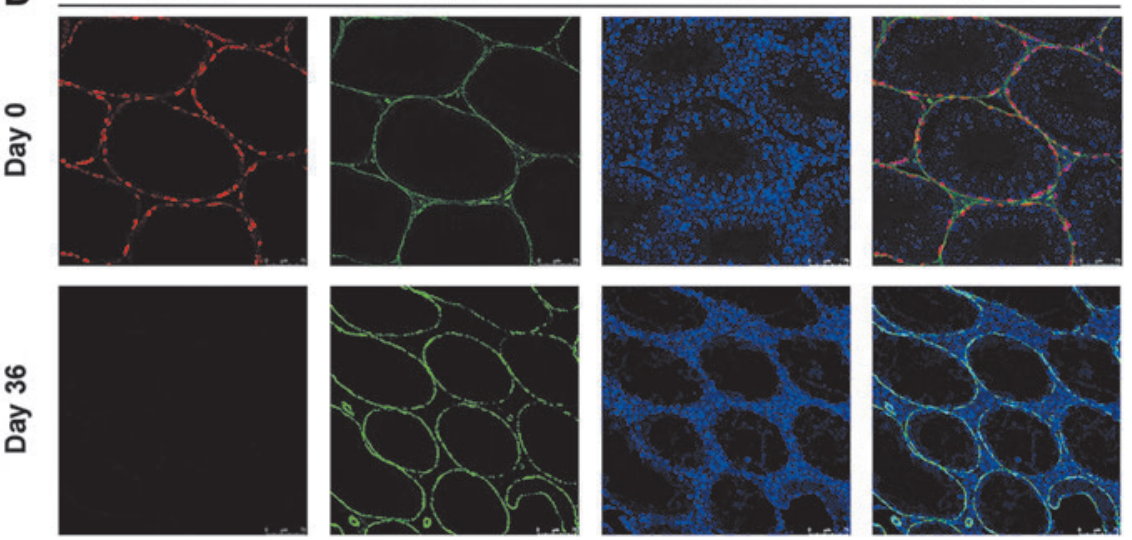

and expressed the zinc finger and BTB domain containing 16 (PLZF) and UCHL1 (markers of SSCs) (Fig. 3A, B). To confirm the event of necroptosis in SSCs, we used TSZ, which is a necroptosis inducer to treat the SSCs. After TSZ treatment for $8 \mathrm{~h}$, the number of SSCs was declined obviously when compared with the PBS or DMSO group evaluated by MTS assays $(P<0.05, n=3)$, while the number of cells in the MEF group did not change obviously $(P>0.05$, $n=3$ ) (Fig. 3C). Furthermore, the protein expression of pMLKL was upregulated at $8 \mathrm{~h}$ after TSZ treatment $(P<0.01$ vs. PBS or DMSO group, $n=6)$, whereas the expression level of RIPK1 was significantly downregulated, confirmed by Western blotting analysis $(P<0.01$ vs. PBS or DMSO group, $n=6$ ) (Fig. 3D). These data showed that the RIPK1-RIPK3-pMLKL pathway mediates necroptosis in mouse SSCs by TSZ. Moreover, a large number of PLZF 

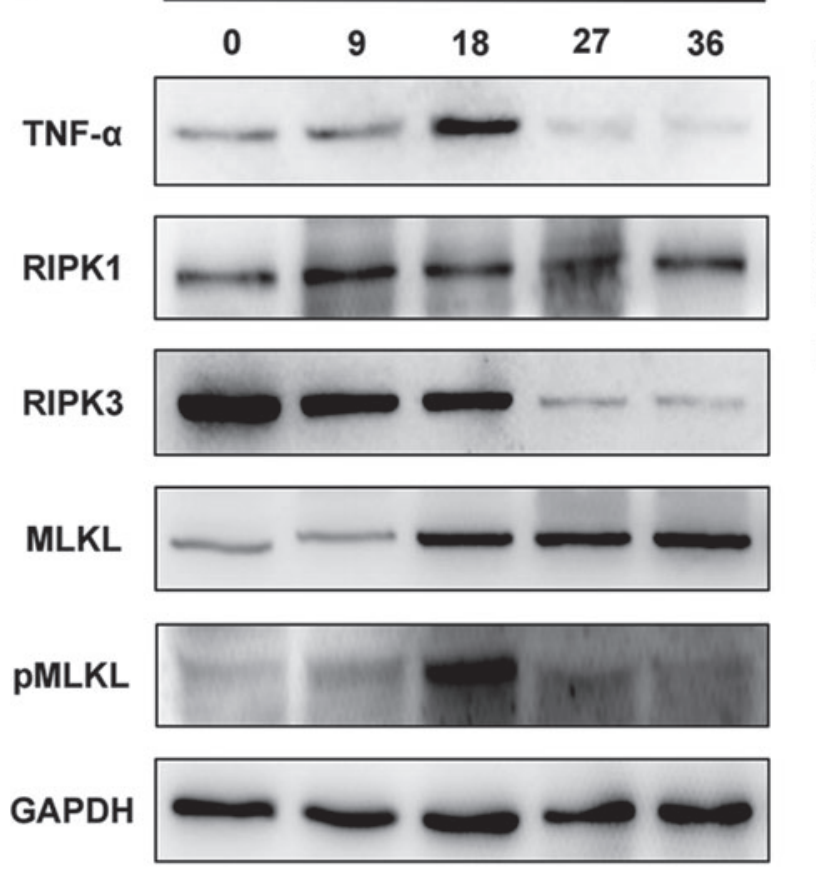

DAPI

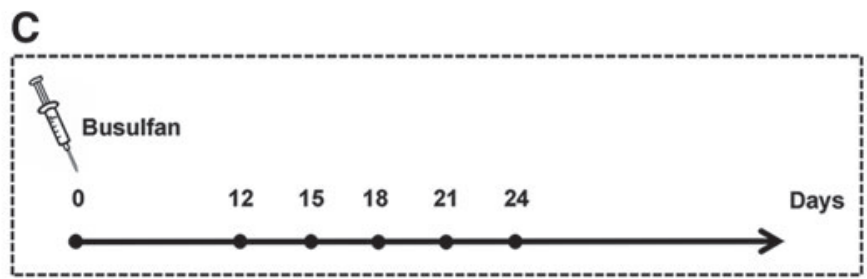

D
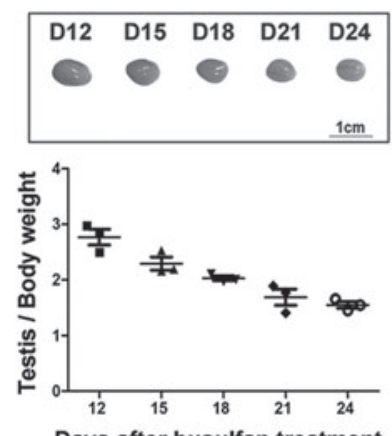

Days after busulfan treatment
E

Days after busulfan treatment

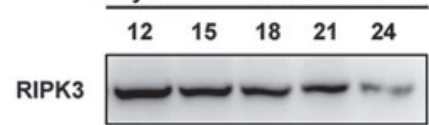

PMLKL

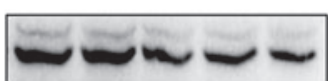

UCHL1

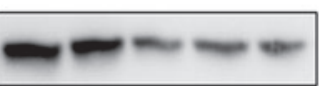

GAPDH

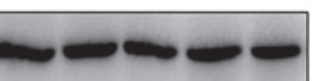

B
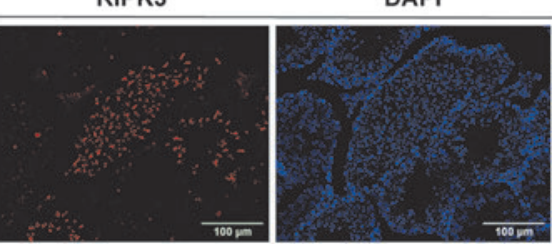

Merge

Merge
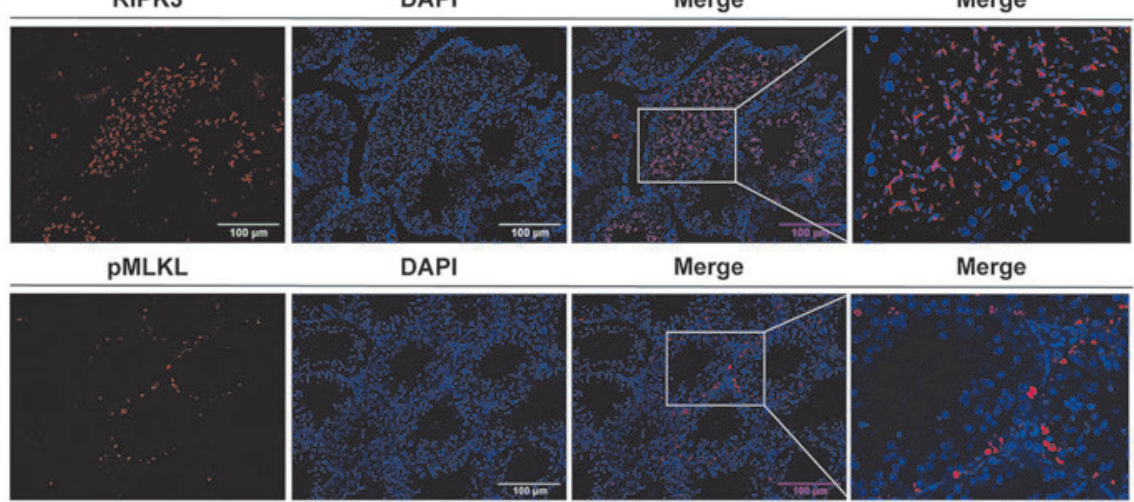

DAPI

Merge

Merge
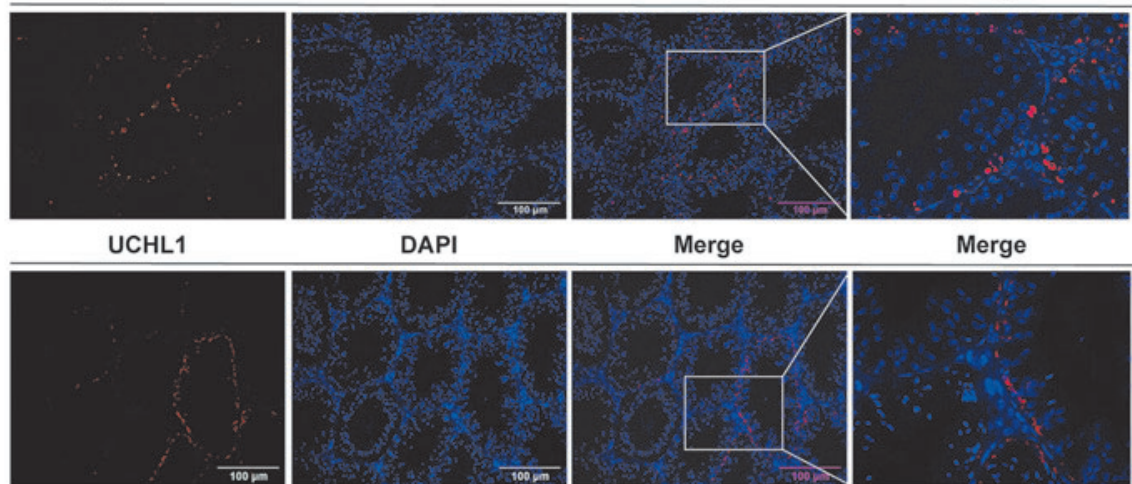

DAPI

Merge

Merge
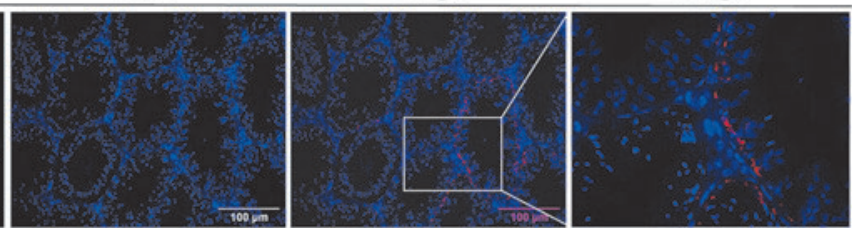

Negative

DAPI

Merge

Merge
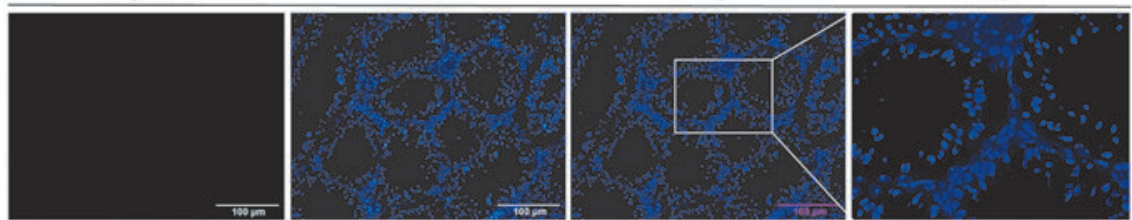

FIG. 2. The necroptosis of SSCs was closely related to the busulfan-induced gonadotoxicity in mice. (A) The kinetic expression of necroptosis components (TNF- $\alpha$, RIPK1, RIPK3, MLKL, and pMLKL) in testes after busulfan treatment. Western blotting analysis showed the level of pMLKL was increased dramatically on day 18. (B) Immunofluorescent staining of RIPK3, pMLKL, and UCHL1 in testes after busulfan treatment on day 18. Scale bar $=100 \mu \mathrm{m}$. (C) The scheme of intraperitoneal injections of busulfan and sample collection time points to explore the relationship between necroptosis and SSCs. ( $n=3$ per group). (D) The ratio of testis weight to body weight after busulfan treatment showed a declining trend ( $n=3$ per group). (E) The protein expression of RIPK3, pMLKL, and UCHL1 in testes after busulfan treatment from day 12 to 24. MLKL, mixed lineage kinase domain like; pMLKL, phosphorylated MLKL; RIPK, receptor-interacting protein kinase; SSC, spermatogonial stem cell; TNF- $\alpha$, tumor necrosis factor-alpha. 
A
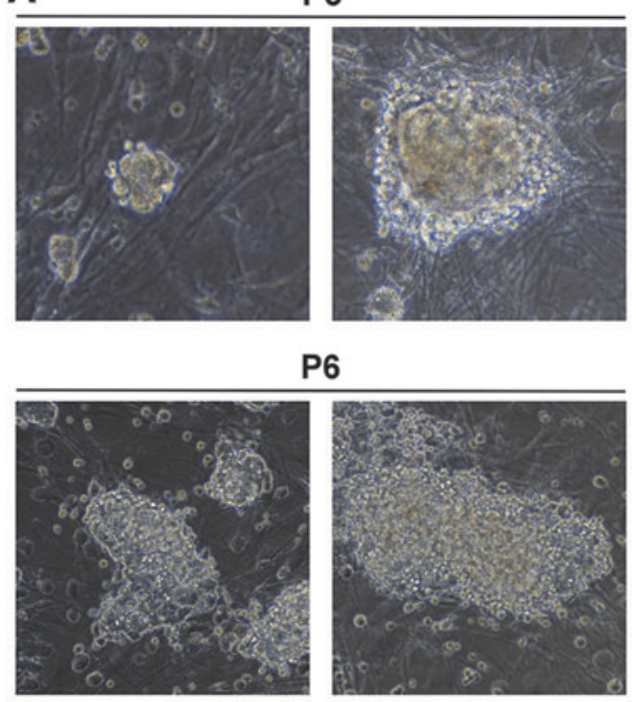

B
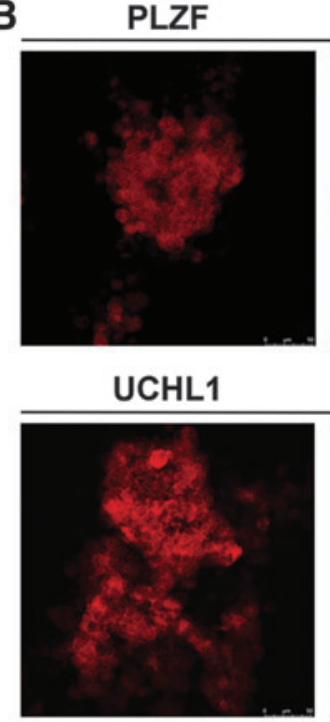

DAPI

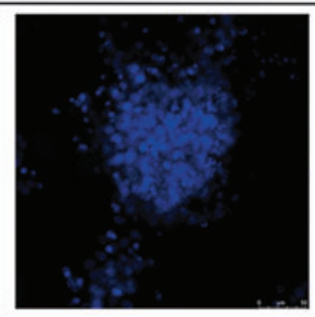

DAPI
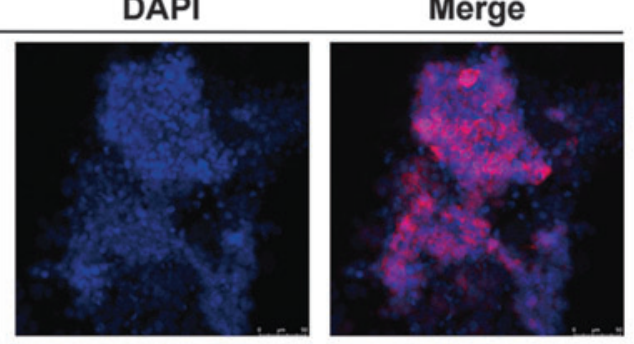

C

MEF

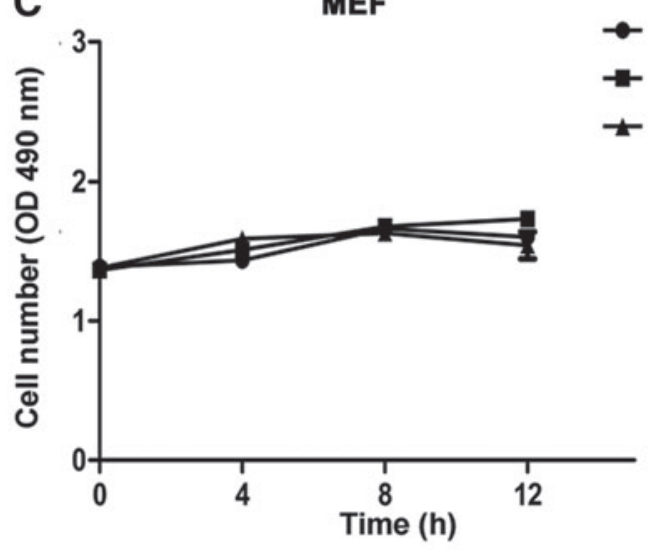

D
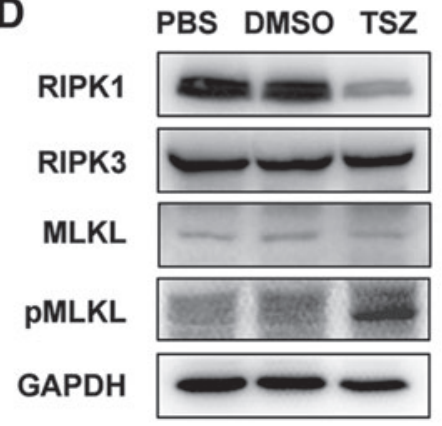

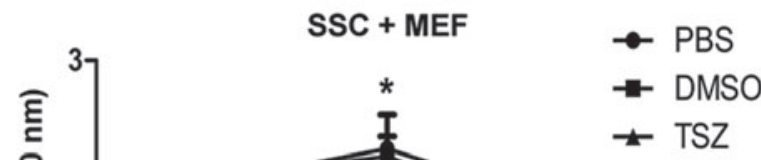

PBS

DMSO TSZ

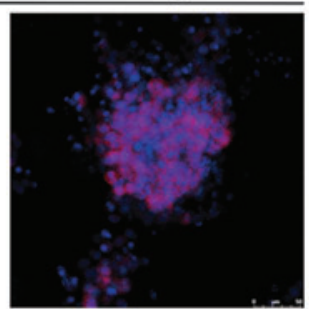

Merge

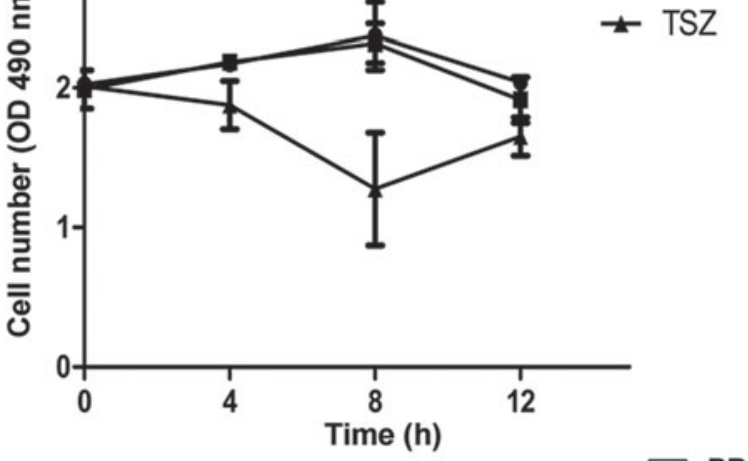

PBS

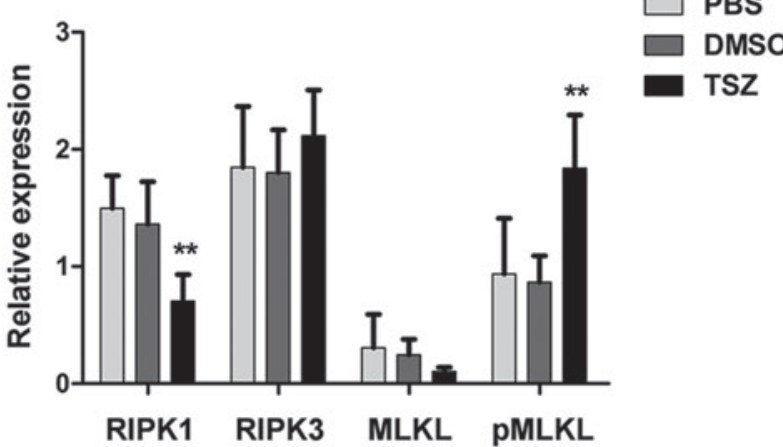

FIG. 3. The RIPK1-RIPK3-pMLKL pathway mediates necroptosis in mouse SSCs. (A) Morphology of SSCs in different passage (P3 and P6) at $200 \times$ field. (B) Immunofluorescent staining of PLZF and UCHL1 to identify the SSCs. Scale bar $=50 \mu \mathrm{m}$. (C) The MTS assays measured the SSC proliferation after treatment by the necroptosis inducer TSZ. MEF was the control group. The number of SSCs was declined obviously after TSZ treatment at $8 \mathrm{~h}(* P<0.05$ vs. PBS or DMSO, $n=3$ per group). (D) The protein expression of pMLKL in SSCs was upregulated at $8 \mathrm{~h}$ after TSZ treatment $(* * P<0.01$ vs. PBS or DMSO, $n=6$ per group). (E) Morphology of SSCs after PBS, DMSO, or busulfan treatment at $200 \times$ field. Most of the SSCs were destroyed by the busulfan at $24 \mathrm{~h}$. (F) The CCK-8 assays showed the number of MEFs was not obviously changed after busulfan treatment at $24 \mathrm{~h}(P>0.05$ vs. PBS or DMSO, $n=5$ per group). (G) Immunofluorescent staining of PLZF after PBS, DMSO, or busulfan treatment at $24 \mathrm{~h}$. Scale bar $=50 \mu \mathrm{m}$. (H) Double immunofluorescence staining of pMLKL and PLZF after busulfan treatment at $24 \mathrm{~h}$. Scale bar $=20 \mu \mathrm{m}$. (I) The protein expression of pMLKL was downregulated after busulfan treatment, while increased after suppressing the necroptosis of SSCs by RIPA-56 in busulfantreated SSCs. (J) The CCK-8 assays showed the number of SSCs was increased after RIPA-56 treatment in busulfan-treated SSCs at $24 \mathrm{~h}(* * P<0.01$ vs. BU, $n=5$ per group). BU, busulfan; CCK-8, cell counting kit-8; DMSO, dimethyl sulfoxide; MEF, mouse embryonic fibroblast; PBS, phosphate-buffered saline; TSZ, TNF- $\alpha$, SM-164 and Z-VAD-FMK. 


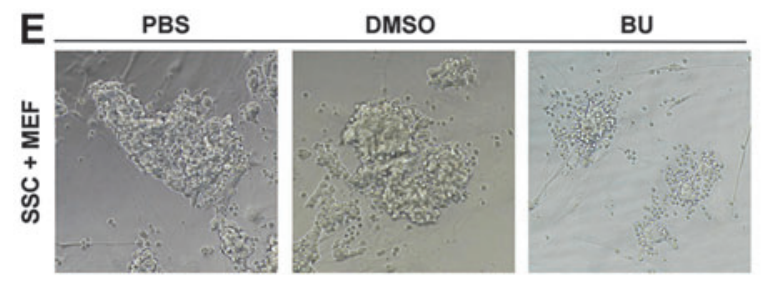

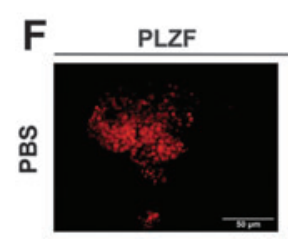
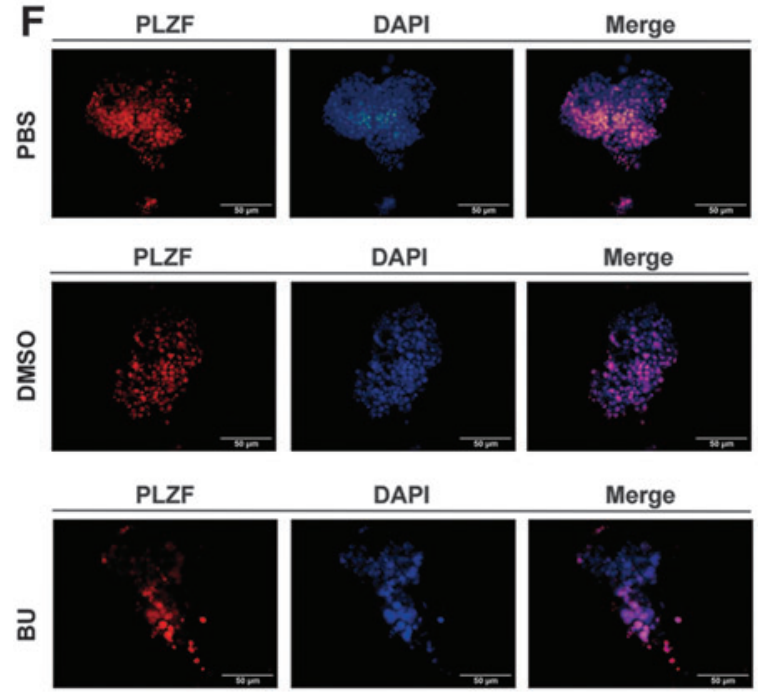

Merge
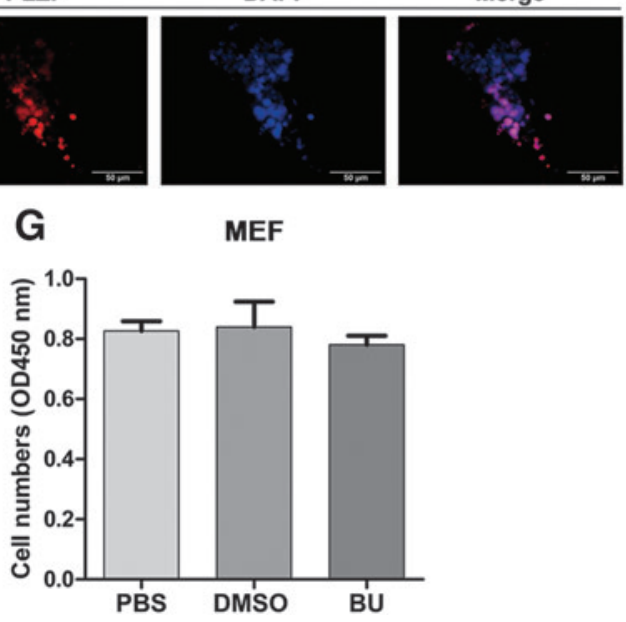

H

ฉั
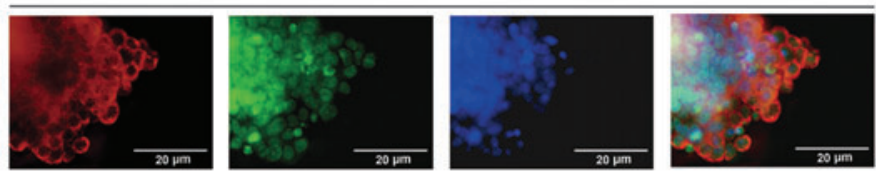

I PBS DMSO BU RIPA-56 BU+RIPA-56

PMLKL

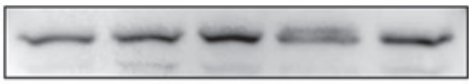

GAPDH

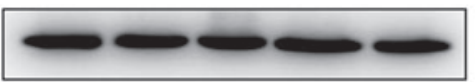

$\mathbf{J}$

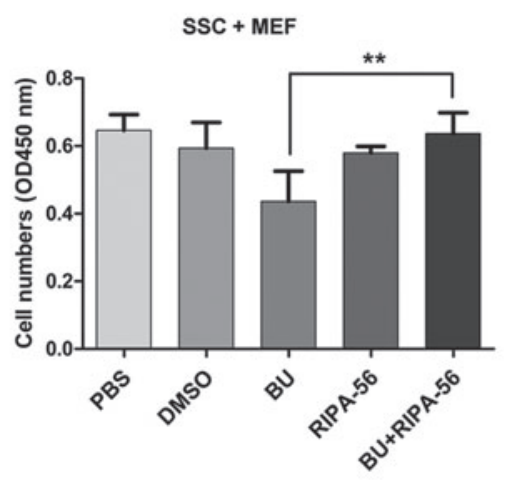

FIG. 3. (Continued).

SSCs were shrunk or deformed, and then died by busulfan administration at $24 \mathrm{~h}$ (Fig. 3E, F), whereas the number of MEFs was not obviously changed $(P>0.05$ vs. PBS or DMSO, $n=5$ ) (Fig. 3G). Double immunofluorescence staining showed that pMLKL co-stain with PLZF in busulfan-treated SSCs at $24 \mathrm{~h}$ (Fig. $3 \mathrm{H})$. Interestingly, after administration of RIPA-56, which is a necroptosis inhibitor, in busulfan-treated SSCs for $24 \mathrm{~h}$, the pMLKL protein expression was downregulated and the survival number of SSCs was increased $(P<0.01$ vs. busulfan, $n=5)$ (Fig. 3I, J). Taken together, these results demonstrated that the RIPK1RIPK3-pMLKL pathway mediates necroptosis in mouse SSCs treated by busulfan.

\section{The inhibitory action of RIPA-56 on necroptosis on day 18 in busulfan-induced mice}

To determine whether RIPK1 could be a potential target for inhibiting necroptosis in vivo, we examined the inhibi- tory action of RIPA-56 on day 18 in busulfan-induced mice to design a reasonable plan $(n=3)$ (Fig. 4A). After daily intragastric administration of RIPA-56 for different time periods, the levels of pMLKL expression in groups 0-9, 9-18, and 0-18 (administration of RIPA-56 daily in busulfaninduced mice from day 0 to 9 , day 9 to 18 , or day 0 to 18 , respectively) were all reduced when compared with the model group (mice with busulfan only) (Fig. 4B). After administration of RIPA-56 from day 0 to 18 , the immunofluorescence staining showed that there was nearly no staining in group 0 18 , and the number of $\mathrm{pMLKL}^{+}$cells per tubule was obviously decreased when compared with the model group $(P<0.001$, $n=3$ ). (Fig. 4C, D). These results indicated that necroptosis was inhibited in the testes of busulfan-induced mice by the suppression of RIPK1 with RIPA-56, and continual administration of RIPA-56 elicited maximal inhibitory effects. Hence, we adopted daily intragastric administration of RIPA-56 for 36 days after busulfan treatment, which was just beyond one seminiferous epithelium cycle, in the following experiment. 
FIG. 4. The inhibitory action of RIPA-56 on necroptosis on day 18 in busulfan-induced mice. (A) The scheme of intraperitoneal injections of busulfan and daily intragastric administration of RIPA-56 to examine the inhibitory action of RIPA-56. (B) The protein expression of pMLKL was downregulated evidently by intragastric administration of RIPA-56 from day 0 to 18 . (C) Immunofluorescent staining of pMLKL in different groups. Scale bar $=100 \mu \mathrm{m}$. (D) After administration of RIPA-56 from day 0 to 18 , the number of $\mathrm{pMLKL}^{+}$cells per tubule was declined when compared with the model group (*** $P<0.001, n=3$ per group). Model: busulfan treatment only; Normal: group without treatment; $0-9,9-18$, or $0-18$ : intragastric administration of RIPA-56 daily in busulfan-induced mice from day 0 to 9 , day 9 to 18 , or day 0 to 18 , respectively.
The cytoprotection of SSCs and restoration of spermatogenesis on day 36 after RIPA-56 treatment in busulfan-induced mice

To understand the cytoprotection of SSCs and restoration of spermatogenesis, we used the busulfan-induced mice.

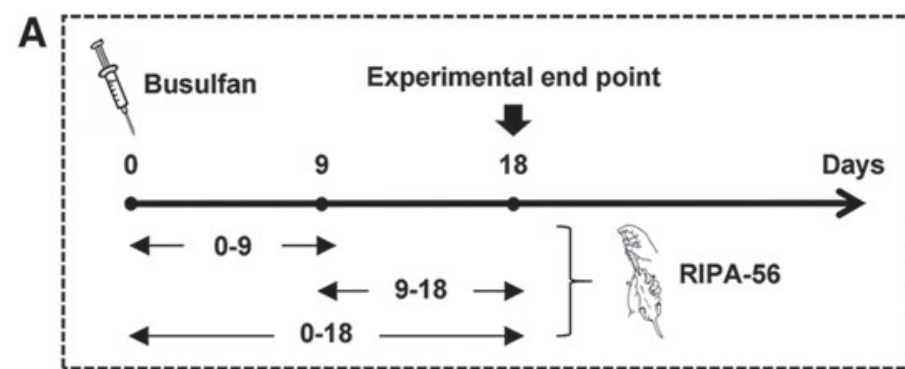

B

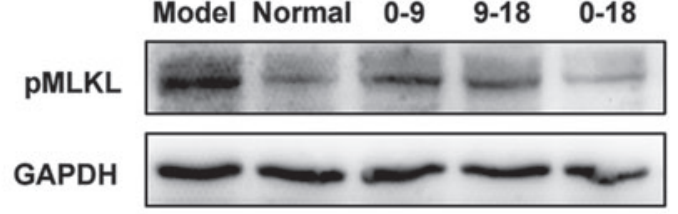

C
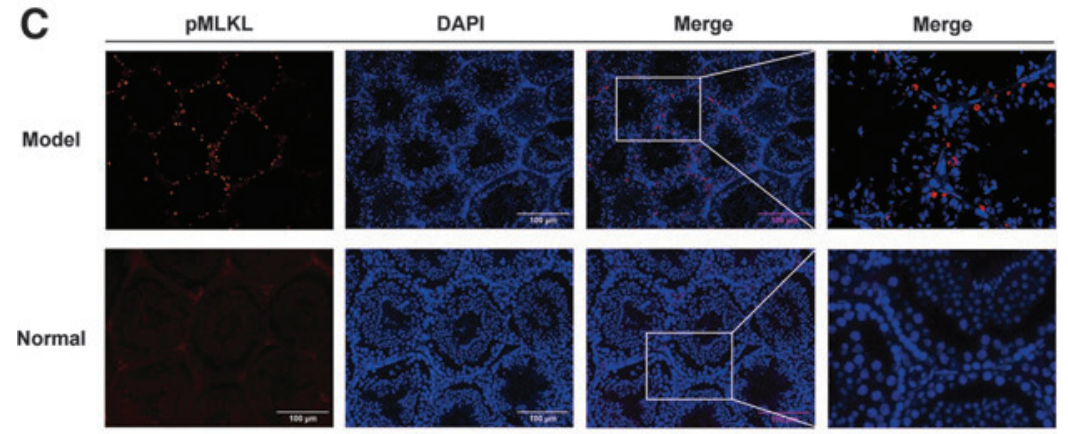

$0-9$
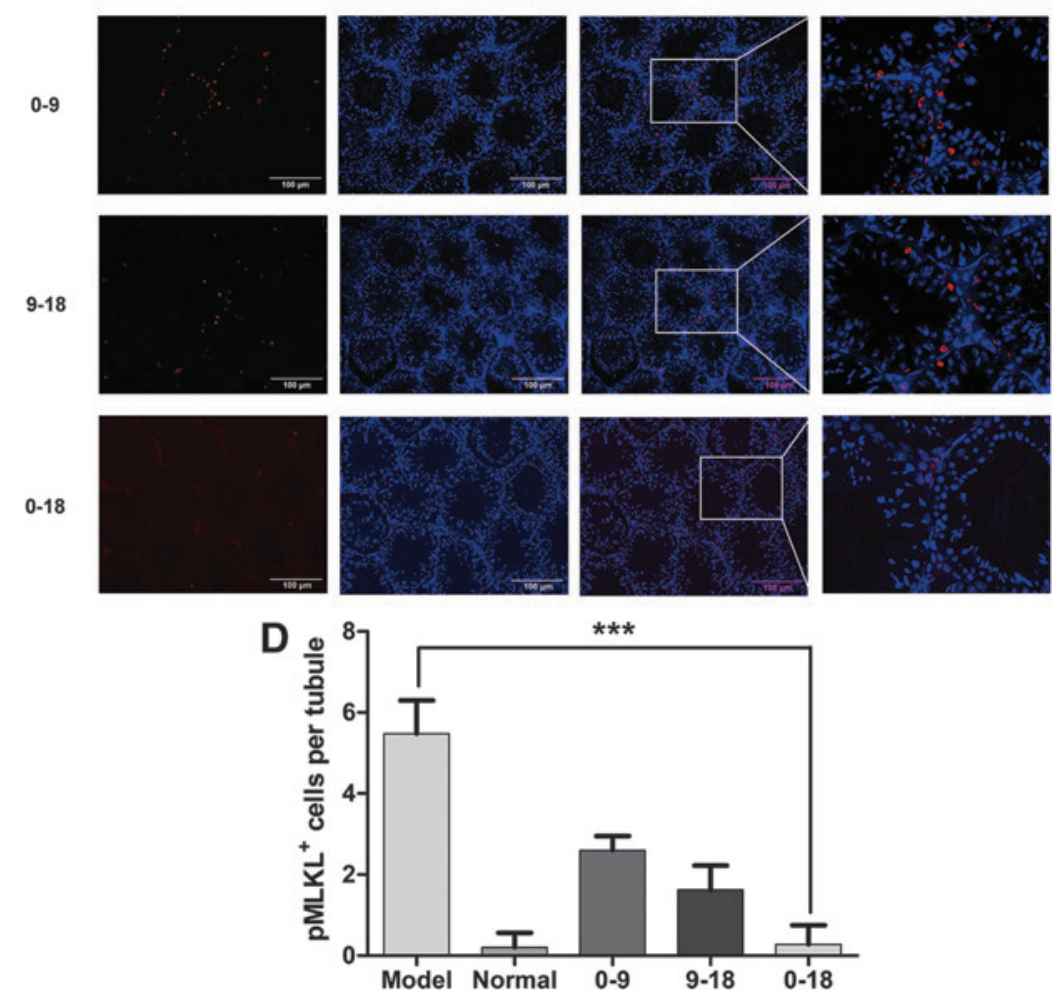

After daily intragastric administration of RIPA-56 for 36 days, the ratio of testis weight to body weight was increased in the RIPA-56 group compared with the Model group $(P<0.05, n=6)$, while there was no notable change in the Vehicle group compared with the Model group $(P>0.05, n=6)$, indicating alleviation of spermatogenic cell 


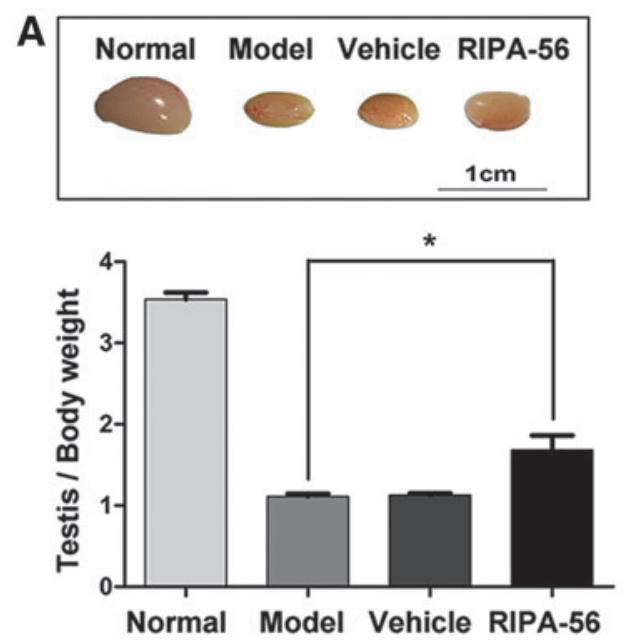

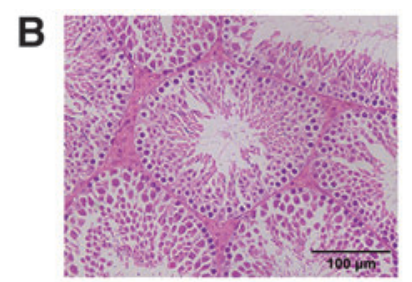

Normal

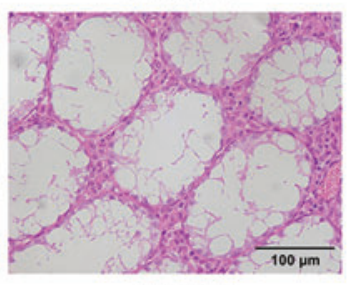

Vehicle

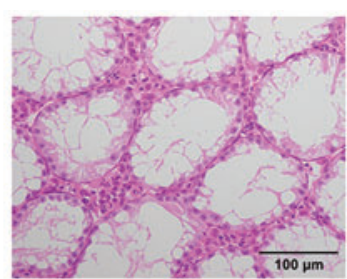

Model

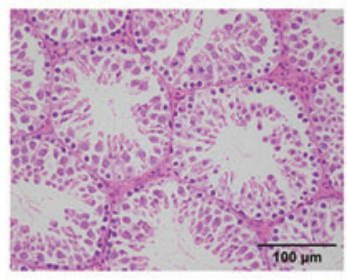

RIPA-56
C

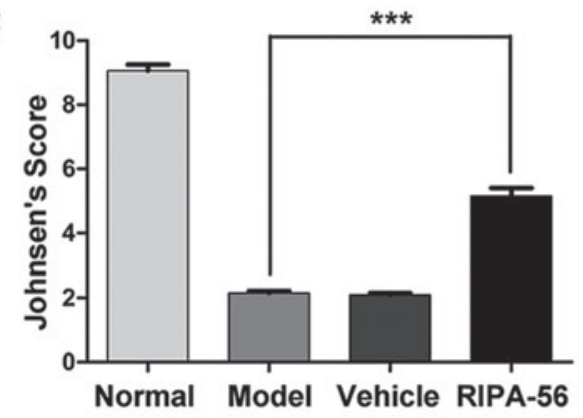

E

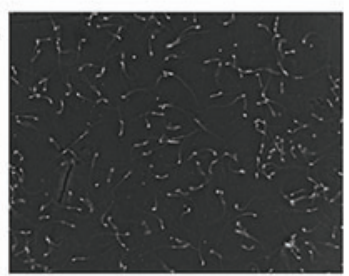

Normal

F

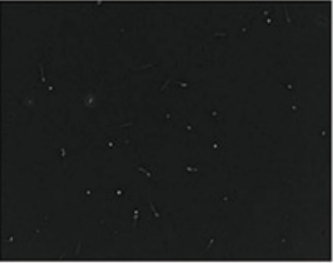

Model

D

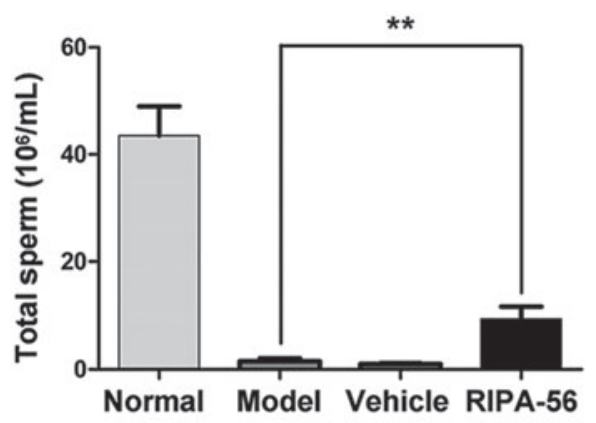

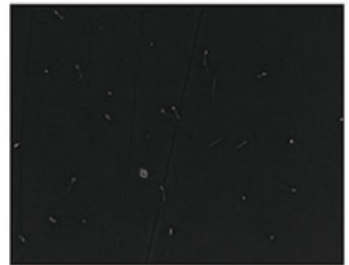

Vehicle

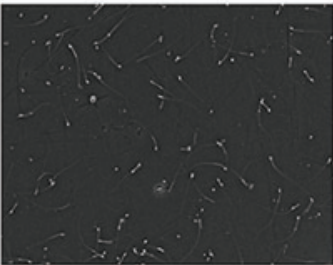

RIPA-56

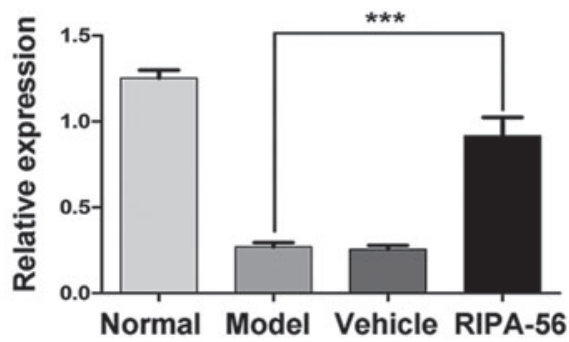

FIG. 5. The cytoprotection of SSCs and restoration of spermatogenesis on day 36 after RIPA-56 treatment in busulfaninduced mice. (A) The ratio of testis weight to body weight showed an increase in the RIPA-56 group and decline in the model group $(* P<0.05$ vs. model, $n=6$ per group). (B, C) H\&E staining showed the RIPA-56 group had a considerable number of spermatogenic cells in seminiferous tubules. The Johnsen's score revealed that the score was increased in the RIPA-56 group $(* * * P<0.001$ vs. model, $n=6$ per group). Scale bar $=100 \mu \mathrm{m}$. (D, E) The epididymal spermatozoa analysis showed a considerable number of spermatozoa (white points) in the cauda epididymidis in the RIPA-56 group under microscopy analysis at $400 \times$ magnification. The total spermatozoa count in the RIPA-56 group was improved $(* * P<0.01$ vs. model, $n=3$ per group). (F, G) Western blotting analysis and immunofluorescent staining demonstrated that the expression of UCHL1 was increased in the RIPA-56 group $(* * * P<0.001$ vs. model, $n=6$ per group). Scale bar $=100 \mu \mathrm{m}$. (H) The number of $\mathrm{UCHL}^{+}$cells per tubule in four groups by the immunofluorescent staining ( $n=6$ per group). Vehicle or RIPA-56: intragastric administration of PBS+DMSO or RIPA-56 daily in busulfan-induced mice from day 0 to 36 , respectively. 

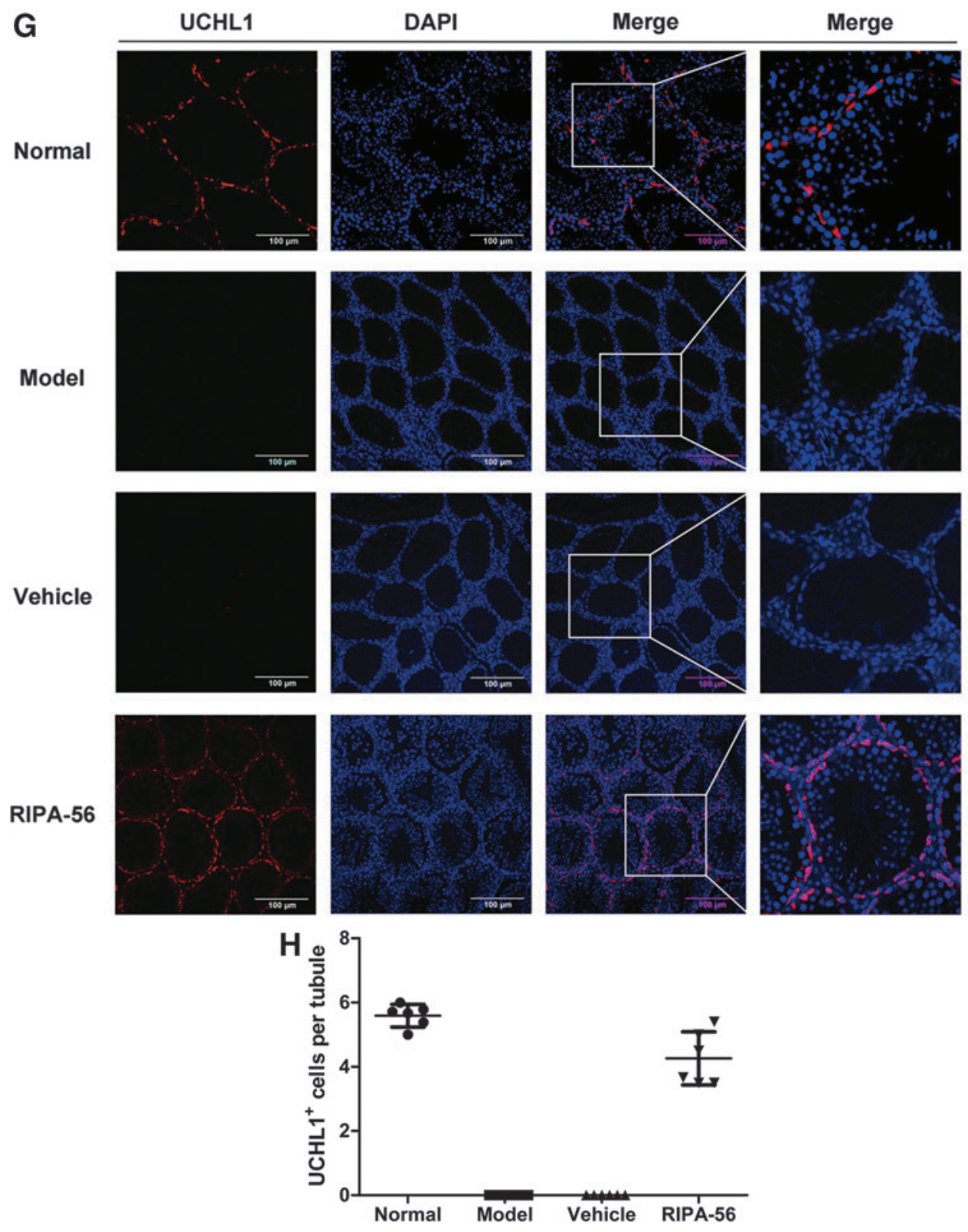

FIG. 5. (Continued).

loss after treatment (Fig. 5A). H\&E staining revealed that a considerable number of spermatogenic cells remained in seminiferous tubules in the RIPA-56 group compared with the Model group, despite frequent disarrangement (Fig. 5B). Meanwhile, the Johnsen's score in the RIPA-56 group was increased compared with those in the Model group $(P<0.001, n=6)$, suggesting that RIPA-56 leads to obvious spermatogenesis protection (Fig. 5C). In addition, the epididymal spermatozoa analysis showed that total spermatozoa count in cauda epididymidis was restored in mice that received 36 days of RIPA-56 treatment $(P<0.05, n=3)$ (Fig. 5D, E). More importantly, the expression of UCHL1 was higher in the RIPA-56 group than in the Model group $(P<0.001, n=6)$, but there was no obvious alteration in the Vehicle group compared with the Model group $(P>0.05$, $n=6$ ) (Fig. 5F). Immunofluorescence staining also dem- onstrated that the RIPA-56 group presented an alleviation of $\mathrm{UCHL}^{+} \mathrm{SSC}$ loss compared with the Model group on day 36 (Fig. 5G, H). These results clearly showed the therapeutic effects of RIPK1 inhibition in protecting SSCs and spermatogenic function from mice with busulfaninduced gonadotoxicity.

\section{Discussion}

In this study, we first demonstrated that RIPA-56, an RIPK1 inhibitor that can inhibit necroptosis, could be a novel agent to preserve fertility in male mice with busulfaninduced gonadotoxicity. RIPK1-driven necroptosis, especially in SSCs of testes, was associated with busulfaninduced gonadotoxicity. After suppressing RIPK1 with RIPA-56 over a spermatogenic cycle, the ratio of testis 
weight to body weight, quantity of SSCs, spermatogenesis score, and number of epididymal spermatozoa were all improved, indicating a series of beneficial effects from targeting necroptosis.

Chemotherapy and irradiation are designed to target neoplastic cells. However, they are highly detrimental to cells and have numerous side effects on male spermatogenesis and fertility by causing dysregulation of cellular metabolism, protein dysfunction, genetic damage, and cellular death [29]. For example, busulfan, a classic alkylating agent, is widely used for allogeneic hematopoietic progenitor cell transplantation in prepubertal patients with chronic myeloid leukemia, but it damages SSCs and differentiating spermatogonia, and is likely to cause prolonged azoospermia [30,31]. Our previous study demonstrated that busulfan initially induced progressive atrophic lesions, frequent disarrangement of spermatogenic cells, and a thinner seminiferous epithelium in testes, ultimately resulting in the loss of most SSCs and impairment of spermatogenesis [17].

Necroptosis is a form of programmed cell death that plays an important role in regulating the fate of individual cells during embryonic development and adult life in multicellular organisms [32-34]. Recent studies confirm that necroptosis is the mechanism underlying male reproductive system aging. In the testes of old mice, pMLKL, the necroptosis activation marker, was specifically detected in SSCs [21]. We also reported previously that necroptosis occurs in the SSCs of aging human testes with reduced spermatogenic function [35]. In this study, we further demonstrated that necroptosis was associated with the busulfan-induced gonadotoxicity in testes and occurred especially in $\mathrm{UCHL}^{+} \mathrm{SSCs}$.

The activation of necroptosis by ligands of death receptors requires the kinase activity of RIPK1, which is a key druggable target in the necroptosis pathway and mediates the activation of RIPK3 and MLKL [34]. Blocking the kinase activity of RIPK1 allows cell survival and proliferation in the presence of death receptor ligands. Hence, RIPK1 inhibition is a novel therapeutic strategy for human inflammatory and degenerative diseases, including amyotrophic lateral sclerosis and Alzheimer's disease [36,37]. Recent studies reported that feeding of wild-type mice with the RIPK1 inhibitor RIPA-56 before the normal onset of age-related changes in reproductive organs could restore fertility $[21,38]$. Thus, directly inhibiting the necroptosis of SSCs, which are the only stem cells involved in spermatogenesis, may be a novel option to restore male fertility [39].

For adult or pubertal males, sperm or testicular tissue cryopreservation is usually advised when a patient is diagnosed with cancer, regardless of the gonadotoxicity of their followed chemotherapy or radiotherapy, if necessary [40,41]. However, young male cancer survivors usually have little concern about their fertility at the time of cancer diagnosis, but more than half ultimately regret not acting sooner and are interested in fertility preservation during cancer treatment. Importantly, prepubertal boys are not yet producing mature sperm, but do harbor SSCs, and no proven fertility preservation options are currently available. SSC transplantation has been successful in animal models, but this approach is still at the experimental stage for humans $[15,42]$. Cryopreservation of testicular tissue needs to be established by screening residual malignant cells, particu- larly in the case of leukemia or lymphoma [6]. What methods can we offer to prepubertal boys who are suffering from a malignant disease?

In fact, previous studies have reported abundant ideas to ameliorate chemotherapy- or irradiation-induced gonadotoxicity in male cancer patients. Melatonin, an antioxidant agent, was found to successfully ameliorate high concentrations of ROS and p53 by promoting MnSOD and SIRT1 expression in busulfan-induced mice [10]. Moreover, Hermann and colleagues demonstrated that mouse spermatogenesis and undifferentiated spermatogonia could be protected from loss following busulfan treatment with granulocyte colony-stimulating factor because its receptor was expressed on undifferentiated spermatogonia [11,12]. In addition, Xiong and colleagues revealed that injection of human umbilical cord mesenchymal stem cells into the testis of busulfan-induced mice also enhanced germ cellassociated gene expression [43]. However, these methodologies were not able to precisely and effectively protect spermatogenesis before or during gonadotoxic treatment for male cancer patients.

A series of studies have already proven that targeting RIPK1, instead of RIPK3 or MLKL, is a safe and important therapeutic strategy to treat inflammatory and degenerative diseases [44,45]. Compared to known RIPK1 inhibitors, RIPA-56 is a highly potent, selective, and metabolically stable inhibitor with an established $\mathrm{IC}_{50}$ in both human and murine cells in vivo [38]. Fortunately, we found that inhibiting SSC necroptosis with RIPA-56 could ameliorate busulfan-induced gonadotoxicity by improving the ratio of testis weight to body weight, quantity of SSCs, spermatogenesis score, and number of epididymal spermatozoa, thereby relieving the damage to spermatogenesis. Interestingly, based on our data, RIPA-56 partially protected against the effects of ongoing chemotherapy procedures, suggesting that it has utility even when cancer treatment has already started. Overall, a series of beneficial effects were observed upon targeting necroptosis in SSCs of mice undergoing busulfan treatment.

Consistent with the notion of testicular tissue cryopreservation or SSC banking, we insist on the idea that there is hope in the existence of SSCs, the only stem cells involved in spermatogenesis. However, it is equally important that, a feasible and precise methodology capable of protecting spermatogenesis in male cancer patients before or during gonadotoxic treatment is needed. Consequently, we deduced that targeting SSC necroptosis by the oral administration of RIPA-56 could be a potentially novel strategy that can be developed from the bench to the bedside to address male oncofertility, especially for prepubertal boys. Although our study put forward a new potential method that could provide insight into fertility preservation, there are certain potential limitations to consider. This study involved few mating and fertility tests and lacked an exploration of the appropriate dosage of RIPA-56.

Taken together, we have shown that inhibition of RIPK1driven necroptosis of SSCs in mice with busulfan-induced gonadotoxicity could preserve fertility by alleviating SSC loss and spermatogenesis damage. These reassuring data have provided a novel strategy to protect fertility in male cancer survivors, especially the prepubertal boys, who did not cryopreserve sperm or freeze testicular tissue. 


\section{Acknowledgment}

We would like to thank Professor Wenliang Zhou for his assistance on epididymal spermatozoa count.

\section{Author Disclosure Statement}

No competing financial interests exist.

\section{Funding Information}

This work was supported by the National Natural Science Foundation of China (nos. 81671834, 81671449, 81771577, 81873829, 81971314, and 81971759); the Guangdong Special Support Plan-Science and Technology Innovation Youth Top Talents Project (2016TQ03R444); the Science and Technology Planning Project of Guangdong Province (2016B030230001); the Key Scientific and Technological Program of Guangzhou City (201604020189); the Pearl River S\&T Nova Program of Guangzhou (201806010089), and the Youth Teacher Training Project of Sun Yat-sen University (No. 17ykpy68).

\section{Supplementary Material}

Supplementary Table S1

\section{References}

1. Bray F, J Ferlay, I Soerjomataram, RL Siegel, LA Torre and A Jemal. (2018). Global cancer statistics 2018: GLOBOCAN estimates of incidence and mortality worldwide for 36 cancers in 185 countries. CA Cancer J Clin 68:394-424.

2. Zhao H, L Jin, Y Li, C Zhang, R Wang, Y Li, W Huang, C Cui, H Zhang, et al (2019). Oncofertility: what can we do from bench to bedside? Cancer Lett 442:148-160.

3. Moss JL, AW Choi, MK Fitzgerald Keeter and RE Brannigan. (2016). Male adolescent fertility preservation. Fertil Steril 105:267-273.

4. Frydman R and M Grynberg. (2016). Introduction: male fertility preservation: innovations and questions. Fertil Steril 105:247-248.

5. Oktay K, BE Harvey, AH Partridge, GP Quinn, J Reinecke, HS Taylor, WH Wallace, ET Wang and AW Loren. (2018). Fertility preservation in patients with cancer: ASCO Clinical Practice Guideline Update. J Clin Oncol 36:19942001.

6. Kenney LB, Z Antal, JP Ginsberg, BS Hoppe, SL Bober, RN Yu, LS Constine, HM van Santen, R Skinner and DM Green. (2018). Improving male reproductive health after childhood, adolescent, and young adult cancer: progress and future directions for survivorship research. J Clin Oncol 36:2160-2168.

7. Wyns C, M Curaba, B Vanabelle, A Van Langendonckt and J Donnez. (2010). Options for fertility preservation in prepubertal boys. Hum Reprod Update 16:312-328.

8. Shetty G and ML Meistrich. (2005). Hormonal approaches to preservation and restoration of male fertility after cancer treatment. J Natl Cancer Inst Monogr 36-39.

9. Suomalainen L, JK Hakala, V Pentikainen, M Otala, K Erkkila, MO Pentikainen and L Dunkel. (2003). Sphingosine-1-phosphate in inhibition of male germ cell apoptosis in the human testis. J Clin Endocrinol Metab 88: $5572-5579$.
10. Li B, X He, M Zhuang, B Niu, C Wu, H Mu, F Tang, Y Cui, W Liu, et al. (2018). Melatonin ameliorates busulfaninduced spermatogonial stem cell oxidative apoptosis in mouse testes. Antioxid Redox Signal 28:385-400.

11. Kotzur T, R Benavides-Garcia, J Mecklenburg, JR Sanchez, M Reilly and BP Hermann. (2017). Granulocyte colony-stimulating factor (G-CSF) promotes spermatogenic regeneration from surviving spermatogonia after high-dose alkylating chemotherapy. Reprod Biol Endocrinol 15:7.

12. Benavides-Garcia R, R Joachim, NA Pina, KN Mutoji, MA Reilly and BP Hermann. (2015). Granulocyte colonystimulating factor prevents loss of spermatogenesis after sterilizing busulfan chemotherapy. Fertil Steril 103:270280.e8.

13. Carmely A, D Meirow, A Peretz, M Albeck, B Bartoov and B Sredni. (2009). Protective effect of the immunomodulator AS101 against cyclophosphamide-induced testicular damage in mice. Hum Reprod 24:1322-1329.

14. Neuhaus N and S Schlatt. (2019). Stem cell-based options to preserve male fertility. Science 363:1283-1284.

15. Fayomi AP, K Peters, M Sukhwani, H Valli-Pulaski, G Shetty, ML Meistrich, L Houser, N Robertson, V Roberts, et al. (2019). Autologous grafting of cryopreserved prepubertal rhesus testis produces sperm and offspring. Science 363:1314-1319.

16. Skinner R, RL Mulder, LC Kremer, MM Hudson, LS Constine, E Bardi, A Boekhout, A Borgmann-Staudt, MC Brown, et al. (2017). Recommendations for gonadotoxicity surveillance in male childhood, adolescent, and young adult cancer survivors: a report from the International Late Effects of Childhood Cancer Guideline Harmonization Group in collaboration with the PanCareSurFup Consortium. Lancet Oncol 18:e75-e90.

17. Xie Y, CC Deng, B Ouyang, LY Lv, JH Yao, C Zhang, HC Chen, XY Li, XZ Sun, CH Deng and GH Liu. (2019). Establishing a nonlethal and efficient mouse model of male gonadotoxicity by intraperitoneal busulfan injection. Asian J Androl. DOI: 10.4103/aja.aja_41_19

18. Deng C, Y Xie, C Zhang, B Ouyang, H Chen, L Lv, J Yao, X Liang, Y Zhang, et al. (2019). Urine-derived stem cells facilitate endogenous spermatogenesis restoration of busulfan-induced non-obstructive azoospermic mice by paracrine exosomes. Stem Cells Dev 28:1322-1333.

19. Shan B, H Pan, A Najafov and J Yuan. (2018). Necroptosis in development and diseases. Genes Dev 32:327-340.

20. Weinlich R, A Oberst, HM Beere and DR Green. (2017). Necroptosis in development, inflammation and disease. Nat Rev Mol Cell Biol 18:127-136.

21. Li D, L Meng, T Xu, Y Su, X Liu, Z Zhang and X Wang. (2017). RIPK1-RIPK3-MLKL-dependent necrosis promotes the aging of mouse male reproductive system. Elife 6. DOI: $10.7554 /$ eLife. 27692

22. de Rooij DG. (2017). The nature and dynamics of spermatogonial stem cells. Development 144:3022-3030.

23. Mizuno K, Y Hayashi, Y Kojima, S Kurokawa, S Sasaki and K Kohri. (2007). Influence for testicular development and histological peculiarity in the testes of flutamideinduced cryptorchid rat model. Int J Urol 14:67-72.

24. Wu Y, H Zhou, X Fan, Y Zhang, M Zhang, Y Wang, Z Xie, M Bai, Q Yin, et al. (2015). Correction of a genetic disease by CRISPR-Cas9-mediated gene editing in mouse spermatogonial stem cells. Cell Res 25:67-79.

25. Dong L, M Gul, S Hildorf, SE Pors, SG Kristensen, ER Hoffmann, D Cortes, J Thorup and CY Andersen. (2019). 
Xeno-free propagation of spermatogonial stem cells from infant boys. Int J Mol Sci 20. DOI: 10.3390/ijms20215390

26. Hou J, M Niu, L Liu, Z Zhu, X Wang, M Sun, Q Yuan, S Yang, W Zeng, et al. (2015). Establishment and characterization of human germline stem cell line with unlimited proliferation potentials and no tumor formation. Sci Rep 5:16922.

27. Baert Y, A Braye, RB Struijk, AM van Pelt and E Goossens. (2015). Cryopreservation of testicular tissue before long-term testicular cell culture does not alter in vitro cell dynamics. Fertil Steril 104:1244-1252.e1-e4.

28. Valli H, M Sukhwani, SL Dovey, KA Peters, J Donohue, CA Castro, T Chu, GR Marshall and KE Orwig. (2014). Fluorescence- and magnetic-activated cell sorting strategies to isolate and enrich human spermatogonial stem cells. Fertil Steril 102:566-580.e7.

29. Rabaca A, M Sousa, MG Alves, PF Oliveira and R Sa. (2015). Novel Drug Therapies for Fertility preservation in men undergoing chemotherapy: clinical relevance of protector agents. Curr Med Chem 22:3347-3369.

30. Puyo S, D Montaudon and P Pourquier. (2014). From old alkylating agents to new minor groove binders. Crit Rev Oncol Hematol 89:43-61.

31. Bucci LR and ML Meistrich. (1987). Effects of busulfan on murine spermatogenesis: cytotoxicity, sterility, sperm abnormalities, and dominant lethal mutations. Mutat Res 176: 259-268.

32. Lee SB, JJ Kim, SA Han, Y Fan, LS Guo, K Aziz, S Nowsheen, SS Kim, SY Park, et al. (2019). The AMPKParkin axis negatively regulates necroptosis and tumorigenesis by inhibiting the necrosome. Nat Cell Biol 21:940-951.

33. Dondelinger Y, P Hulpiau, Y Saeys, MJM Bertrand and P Vandenabeele. (2016). An evolutionary perspective on the necroptotic pathway. Trends Cell Biol 26:721-732.

34. Zhou W and J Yuan. (2014). Necroptosis in health and diseases. Semin Cell Dev Biol 35:14-23.

35. Xie Y, L Lv, J Yao, C Zhang, H Chen, W Chen, X Liang, X Sun, C Deng and G Liu. (2019). Phosphorylated mixed lineage kinase domain-like protein in human seminal plasma: a potential novel biomarker of spermatogenic function. Andrologia 51:e13310.

36. Xu D, T Jin, H Zhu, H Chen, D Ofengeim, C Zou, L Mifflin, L Pan, P Amin, et al. (2018). TBK1 suppresses RIPK1-driven apoptosis and inflammation during development and in aging. Cell 174:1477-1491.e19.

37. Caccamo A, C Branca, IS Piras, E Ferreira, MJ Huentelman, WS Liang, B Readhead, JT Dudley, EE Spangenberg, et al. (2017). Necroptosis activation in Alzheimer's disease. Nat Neurosci 20:1236-1246.
38. Ren Y, Y Su, L Sun, S He, L Meng, D Liao, X Liu, Y Ma, C Liu, et al. (2017). Discovery of a highly potent, selective, and metabolically stable inhibitor of receptor-interacting protein 1 (RIP1) for the treatment of systemic inflammatory response syndrome. J Med Chem 60:972-986.

39. Kanatsu-Shinohara M and T Shinohara. (2013). Spermatogonial stem cell self-renewal and development. Annu Rev Cell Dev Biol 29:163-187.

40. Goossens E, D Van Saen and H Tournaye. (2013). Spermatogonial stem cell preservation and transplantation: from research to clinic. Hum Reprod 28:897-907.

41. Menon S, N Rives, N Mousset-Simeon, L Sibert, JP Vannier, S Mazurier, L Masse, V Duchesne and B Mace. (2009). Fertility preservation in adolescent males: experience over 22 years at Rouen University Hospital. Hum Reprod 24:37-44.

42. Wallace WH, RA Anderson and DS Irvine. (2005). Fertility preservation for young patients with cancer: who is at risk and what can be offered? Lancet Oncol 6:209-218.

43. Yang RF, TH Liu, K Zhao and CL Xiong. (2014). Enhancement of mouse germ cell-associated genes expression by injection of human umbilical cord mesenchymal stem cells into the testis of chemical-induced azoospermic mice. Asian J Androl 16:698-704.

44. Ofengeim D, S Mazzitelli, Y Ito, JP DeWitt, L Mifflin, C Zou, S Das, X Adiconis, H Chen, et al. (2017). RIPK1 mediates a disease-associated microglial response in Alzheimer's disease. Proc Natl Acad Sci U S A 114:E8788E8797.

45. Ofengeim D, Y Ito, A Najafov, Y Zhang, B Shan, JP DeWitt, J Ye, X Zhang, A Chang, et al. (2015). Activation of necroptosis in multiple sclerosis. Cell Rep 10:18361849.

Address correspondence to: Dr. Guihua Liu Reproductive Centre The Sixth Affiliated Hospital Sun Yat-sen University Guangzhou 510655

China

E-mail: liuguihua@mail.sysu.edu.cn

Received for publication September 21, 2019

Accepted after revision February 3, 2020

Prepublished on Liebert Instant Online February 5, 2020 